\title{
CORRECTION
}

Xu Wang • Peter Schiavone $(\mathbb{D}$

\section{Correction to: Coulomb force on a line charge in an anisotropic piezoelectric biomaterial}

Published online: 9 December 2021

(C) Springer-Verlag GmbH Germany, part of Springer Nature 2021

Correction to: Continuum Mech. Thermodyn.

$$
\text { https://doi.org/10.1007/s00161-021-01061-9 }
$$

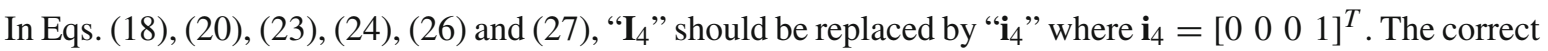
expressions for Eqs. (18), (20), (23), (24), (26) and (27) are then:

$$
\begin{gathered}
\mathbf{f}_{1}(z)=-\frac{q}{2 \pi \mathrm{i}}\left\langle\ln \left(z_{\alpha}-p_{\alpha} d\right)\right\rangle \mathbf{A}_{1}^{T} \mathbf{i}_{4}-\frac{q}{2 \pi \mathrm{i}} \sum_{j=1}^{4}\left\langle\ln \left(z_{\alpha}-\bar{p}_{j} d\right)\right\rangle \mathbf{K}_{j} \overline{\mathbf{A}}_{1}^{T} \mathbf{i}_{4}, \\
F=q E_{2}^{C}(0, d) \\
=\frac{q^{2}}{\pi d} \mathbf{i}_{4}^{T} \operatorname{Im}\left\{\mathbf{A}_{1} \sum_{j=1}^{4}\left\langle\frac{p_{\alpha}}{p_{\alpha}-\bar{p}_{j}}\right\rangle \mathbf{K I}_{j} \overline{\mathbf{A}}_{1}^{T}\right\} \mathbf{i}_{4} \\
=\frac{q^{2}}{\pi d} \mathbf{i}_{4}^{T} \operatorname{Im}\left\{\sum_{j=1}^{4} \sum_{k=1}^{4} \frac{p_{k}}{p_{k}-\bar{p}_{j}} \mathbf{A}_{1} \mathbf{I}_{k} \mathbf{K I}_{j} \overline{\mathbf{A}}_{1}^{T}\right\} \mathbf{i}_{4}, \\
F=\frac{q^{2}}{2 \pi d} \mathbf{i}_{4}^{T} \operatorname{Re}\left\{\sum_{j=1}^{4} \sum_{k=1}^{4} \frac{p_{k}}{p_{k}-\bar{p}_{j}}\left(\mathbf{A}_{1} \mathbf{I}_{k} \mathbf{A}_{1}^{-1}\right) \mathbf{H}_{1}\left(\overline{\mathbf{A}}_{1} \mathbf{I}_{j} \overline{\mathbf{A}}_{1}^{-1}\right)^{T}\right\} \mathbf{i}_{4} \\
-\frac{q^{2}}{\pi d} \mathbf{i}_{4}^{T} \operatorname{Re}\left\{\sum_{j=1}^{4} \sum_{k=1}^{4} \frac{p_{k}}{p_{k}-\bar{p}_{j}}\left(\mathbf{A}_{1} \mathbf{I}_{k} \mathbf{A}_{1}^{-1}\right)(\tilde{\mathbf{H}}+\mathbf{i} \breve{\mathbf{W}})\left(\overline{\mathbf{A}}_{1} \mathbf{I}_{j} \overline{\mathbf{A}}_{1}^{-1}\right)^{T}\right\} \mathbf{i}_{4} .
\end{gathered}
$$

The original article can be found online at https://doi.org/10.1007/s00161-021-01061-9

X. Wang $(\varangle)$

School of Mechanical and Power Engineering, East China University of Science and Technology, 130 Meilong Road, Shanghai 200237, China

E-mail: xuwang@ecust.edu.cn

P. Schiavone $(\varangle)$

Department of Mechanical Engineering, University of Alberta, 10-203 Donadeo Innovation Centre for Engineering, Edmonton, AB T6G 1H9, Canada

E-mail: p.schiavone@ualberta.ca 


$$
\begin{aligned}
F= & \frac{q^{2}}{2 \pi d} \mathbf{i}_{4}^{T} \operatorname{Re}\left\{\sum_{j=1}^{4} \sum_{k=1}^{4} \frac{-\bar{p}_{j}}{p_{k}-\bar{p}_{j}}\left(\mathbf{A}_{1} \mathbf{I}_{k} \mathbf{A}_{1}^{-1}\right) \mathbf{H}_{1}\left(\overline{\mathbf{A}}_{1} \mathbf{I}_{j} \overline{\mathbf{A}}_{1}^{-1}\right)^{T}\right\} \mathbf{i}_{4} \\
& +\frac{q^{2}}{\pi d} \mathbf{i}_{4}^{T} \operatorname{Re}\left\{\sum_{j=1}^{4} \sum_{k=1}^{4} \frac{\bar{p}_{j}}{p_{k}-\bar{p}_{j}}\left(\mathbf{A}_{1} \mathbf{I}_{k} \mathbf{A}_{1}^{-1}\right)(\tilde{\mathbf{H}}+\mathbf{i} \breve{\mathbf{W}})\left(\overline{\mathbf{A}}_{1} \mathbf{I}_{j} \overline{\mathbf{A}}_{1}^{-1}\right)^{T}\right\} \mathbf{i}_{4} . \\
E= & \frac{q}{2} \phi^{C}(0, d)=-\frac{q^{2}}{2 \pi} \mathbf{i}_{4}^{T} \operatorname{Im}\left\{\mathbf{A}_{1} \sum_{j=1}^{4}\left\langle\ln \left[d\left(p_{\alpha}-\bar{p}_{j}\right)\right]\right\rangle \mathbf{K I}_{j} \overline{\mathbf{A}}_{1}^{T}\right\} \mathbf{i}_{4}, \\
F= & -\frac{\partial E}{\partial d}=\frac{q^{2}}{2 \pi d} \mathbf{i}_{4}^{T} \operatorname{Im}\left\{\mathbf{A}_{1} \mathbf{K} \overline{\mathbf{A}}_{1}^{T}\right\} \mathbf{i}_{4}=\frac{q^{2}}{4 \pi d}\left(\mathbf{H}_{1}-2 \tilde{\mathbf{H}}\right)_{44},
\end{aligned}
$$

The final expression for the Coulomb force is correct and remains unchanged.

Publisher's Note Springer Nature remains neutral with regard to jurisdictional claims in published maps and institutional affiliations. 